\title{
The microtubule-associated protein PRC1 is a potential therapeutic target for lung cancer
}

\author{
Steffen Hanselmann ${ }^{1}$, Patrick Wolter ${ }^{1}$, Jonas Malkmus ${ }^{1}$ and Stefan Gaubatz ${ }^{1}$ \\ ${ }^{1}$ Theodor Boveri Institute, Biocenter, University of Wuerzburg and Comprehensive Cancer Center Mainfranken, University of \\ Wuerzburg, University of Wuerzburg, Wuerzburg, Germany \\ Correspondence to: Stefan Gaubatz, email: stefan.gaubatz@biozentrum.uni-wuerzburg.de \\ Keywords: PRC 1; mitotic kinesins; lung adenocarcinoma; therapeutic target \\ Received: August 05, $2017 \quad$ Accepted: December 01, $2017 \quad$ Published: December 22, 2017 \\ Copyright: Hanselmann et al. This is an open-access article distributed under the terms of the Creative Commons Attribution Li- \\ cense 3.0 (CC BY 3.0), which permits unrestricted use, distribution, and reproduction in any medium, provided the original author \\ and source are credited.
}

\section{ABSTRACT}

In this study, we investigated whether proteins that are involved in cytokinesis are potential targets for therapy of lung cancer. We find that the microtubuleassociated protein PRC1 (protein required for cytokinesis 1 ), which plays a key role in organizing anti-parallel microtubule in the central spindle in cytokinesis, is overexpressed in lung cancer cell lines compared to normal cells. Increased expression of PRC1 is correlated with a poor prognosis of human lung adenocarcinoma patients. Lentiviral delivered, inducible RNAi of PRC1 demonstrated that proliferation of lung cancer cell lines strongly depends on PRC1. Significantly, we also show that PRC1 is required for tumorigenesis in vivo using a mouse model for non-small cell lung cancer driven by oncogenic K-RAS and loss of p53. When PRC1 is depleted by in vivo RNA interference, lung tumor formation is significantly reduced. Although PRC1 has been suggested to regulate Wnt/B-catenin signaling in cancer cells, we find no evidence for a role of PRC1 in this pathway in lung cancer. Instead, we show that the depletion of PRC1 results in a strong increase in bi- and multinuclear cells due to defects in cytokinesis. This ultimately leads to apoptosis and senescence. Together these data establish PRC1 as a potential target for therapy of lung cancer.

\section{INTRODUCTION}

Lung cancer is one of the leading causes of malignancy related deaths worldwide. Non-small cell lung cancer (NSCLC) accounts for up to $80 \%$ of all lung cancer cases [1]. The K-RAS oncogene, a small GTPase, is the most frequently mutated gene in NSCLC, with mutations detected in about $30 \%$ of all tumors, mainly of the adenocarcinoma subtype [2]. K-RAS mutation is associated with a poor prognosis of NSCLC and confers resistance to standard therapy. Direct inhibition of K-RAS has been largely unsuccessful and up to date no targeted therapy for oncogenic K-RAS has been developed [3]. Therefore, K-RAS remains an elusive target for cancer therapy and new treatment strategies are needed. One approaches is to inhibit pathways that act downstream or in parallel to activated K-RAS. For example, because oncogenic K-RAS activates signaling through both the PI3K and MEK pathways, several studies have investigated the efficacy of co-targeting the PI3K and MEK pathways with promising results [4, 5]. Interestingly, K-RAS mutant cancer cells also rely on additional pathways that are required indirectly as a consequence of K-RAS pathway activation [6]. These so-called "non-oncogene addiction" pathways represent additional potential targets for therapeutic intervention [7]. For example, it has been demonstrated that K-RAS mutant cells depend on the GATA2 transcription factor or the pro-survival protein $\mathrm{BCL}-\mathrm{X}_{\mathrm{L}}[8,9]$. Interestingly, several proteins with mitotic function such as $\mathrm{APC} / \mathrm{C}$ and the mitotic kinase PLK1 are also selectively required in oncogenic K-RAS-dependent cells [10]. It has been proposed that K-RAS mutant cells experience mitotic alterations termed „mitotic stress" which renders them more sensitive to perturbations in chromosome segregation compared to normal cells $[11,12]$. The increased 
sensitivity of K-RAS mutant cells to inhibition of mitosis is likely a consequence of their high proliferative capacity and increased DNA damage.

Cytokinesis and mitotic exit might be a better cancer therapeutic target than spindle assembly [13, 14]. A key player in cytokinesis is the central spindle, the region of overlapping microtubules in the center of the spindle after the chromosomes have separated in mitosis [15]. The central spindle determines the cleavage plane in anaphase and telophase, it is involved in formation of the actomyosin-based contractile ring that drives cytokinesis and it recruits proteins that mediate abscission, the final step of cell division $[16,17]$. Central spindle formation is mediated by mitotic kinesin motor proteins and by nonmotor microtubule associated protein (MAPs). For the reasons described above, inhibition of these proteins might be a novel strategy for tumor therapy [18].

In this study, we investigated whether proteins involved in central spindle formation are potential targets for the therapy of lung cancer. We show that mitotic kinesins and the microtubule-associated protein PRC1 are overexpressed in lung cancer cell lines. Furthermore, we demonstrate that depletion of PRC1 strongly inhibits the proliferation of five different human lung cancer cell lines due to the induction of apoptosis and senescence. Importantly, we find that PRC1 is required for tumorigenesis in a mouse model for non-small lung cancer driven by oncogenic K-RAS and loss of p53 in vivo. Together these data establish the microtubulebinding protein PRC1 as potential target for therapy of lung cancer.

\section{RESULTS}

\section{Mitotic kinesins and the microtubule-associated protein PRC1 are overexpressed in lung cancer cell lines}

We first analyzed the expression levels of selected mitotic kinesins (KIF4A, KIF14, KIF20A, KIF23, KIF2C, KIFC1) and of the microtubule-binding protein PRC1. KIF4A, KIF14, KIF20A, KIF23 and PRC1 are involved in central spindle formation and cytokinesis $[16,17]$. KIF2C and KIFC1 function in other stages of mitosis but have also been implicated in central spindle function $[19,20]$. These two proteins were included in our study because they have been suggested as potential therapeutic targets [21, 22]. Expression of the kinesins and of PRC1 was analyzed in a panel of human lung cancer cell lines by immunoblotting. Non-transformed BJ and WI-38 fibroblasts were used as a control. Kinesin and PRC1 expression was detected in all cancer cell lines. In contrast, kinesin expression in nontransformed control cells was low (Figure 1A). Highest kinesin expression levels were detected in p53-mutant cell lines compared to cell lines with wildtype p53. KIF23 was already elevated in lung cancer cell lines with wildtype p53-status, suggesting that other pathways contribute to its overexpression in lung cancer cells. mRNA expression of kinesins reflected protein levels with higher levels in p53mutant H23 cells compared to H460 cells with wildtype p53 status (Figure 1B).

\section{Mitotic kinesins and PRC1 are required for proliferation of lung cancer cell lines}

K-RAS mutant tumor cells rely indirectly on a number of survival pathways and experience mitotic stress that has been suggested as a possible target for therapy [10, 11]. To begin to address whether proliferation of K-RAS mutant lung cancer cell lines depends on regulators of the mitotic spindle midzone, we used a multifunctional lentiviral vector to establish stable $\mathrm{H} 23$ and H460 cell lines that express doxycycline-inducible small hairpin RNAs (shRNAs) to deplete mitotic kinesins or PRC1 by RNAi (Figure 2A). The knockdown of mitotic proteins was analyzed by immunoblotting (Figure 2B). Upon doxycycline-induction, the respective targeted protein was specifically downregulated without affecting the expression of other mitotic regulators.

Next, conditional H23 and H460 knockdown cells were plated at low density and treated without or with doxycycline to induce the expression of the shRNA. The number of colonies was determined after 7 days by crystal violet staining (Figure $2 \mathrm{C}$ ). Crystal violet staining was quantified by extraction of the dye and photometric measurement (Figure 2D, 2E). Importantly, when control $\mathrm{H} 23$ and H460 cells were treated with doxycycline, colony formation was not inhibited (Figure 2C). In contrast, depletion of the investigated mitotic regulators inhibited colony formation with different efficiency. Strongest growth inhibitory effects were observed after inhibition of KIF23, KIF4A and PRC1. Depletion of these proteins inhibited the proliferation of $\mathrm{H} 460$ cells (wildtype p53) as well as H23 cells with a p53-mutant status (Figure $2 \mathrm{D}, 2 \mathrm{E})$. The other shRNAs tested had moderate growth inhibitory effects in p53-wildtype cells but were mostly ineffective in p53-mutant cells. That the depletion of KIF23 inhibits proliferation of H460 and H23 cells is consistent with our recent finding that KIF23 is required for proliferation and in vitro and for lung tumor formation in vivo [23].

\section{PRC1 is required for proliferation and cytokinesis of lung cancer cell lines}

In addition to KIF23, depletion of KIF4A and of PRC1 also inhibited proliferation of both $\mathrm{H} 23$ and $\mathrm{H} 460$ cells. We therefore next focused on these two proteins and asked whether they are required for proliferation of additional lung cancer cell lines. We first used the multifunctional lentiviral vector described above to establish additional lung cancer cell lines that stably 
express doxycycline-inducible small hairpin RNAs (shRNAs) directed at KIF4A and PRC1. Although KIF4A was efficiently depleted in HOP62 and HOP92 cell lines after addition of doxycycline (Supplementary Figure 1A), its depletion had only moderate effects on proliferation in these cells (Supplementary Figure 1B, C). Furthermore, the depletion of KIF4A in HOP92 cells did not result in an increase in bi- and multinucleated cells, indicating that it is not required for cytokinesis in these cells (Supplementary Figure 1D). Because KIF4A had only weak effects in these additional lung cancer cell lines, it was not further studied.
We therefore next investigated the effect of depletion of PRC1 in five different human lung cancer cell lines and in immortalized BJ fibroblast cells, which served as an untransformed control cell line. Depletion of PRC1 was first validated by immunoblotting (Figure 3A). We also established control cell lines expressing a shRNA directed at luciferase (shLuc), to exclude possible non-specific effects of doxycycline. Next, conditional knockdown cell lines were plated at low density and treated with different concentrations of doxycycline to induce the expression of the shRNA. Cell number at different time

A

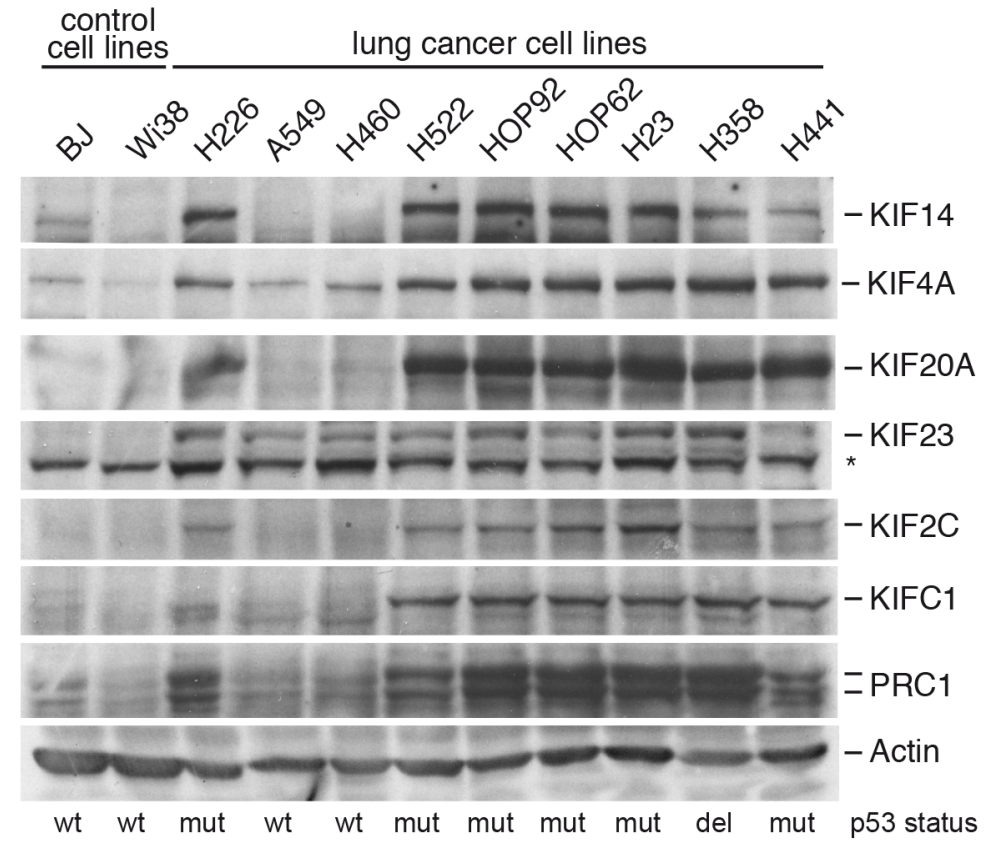

B

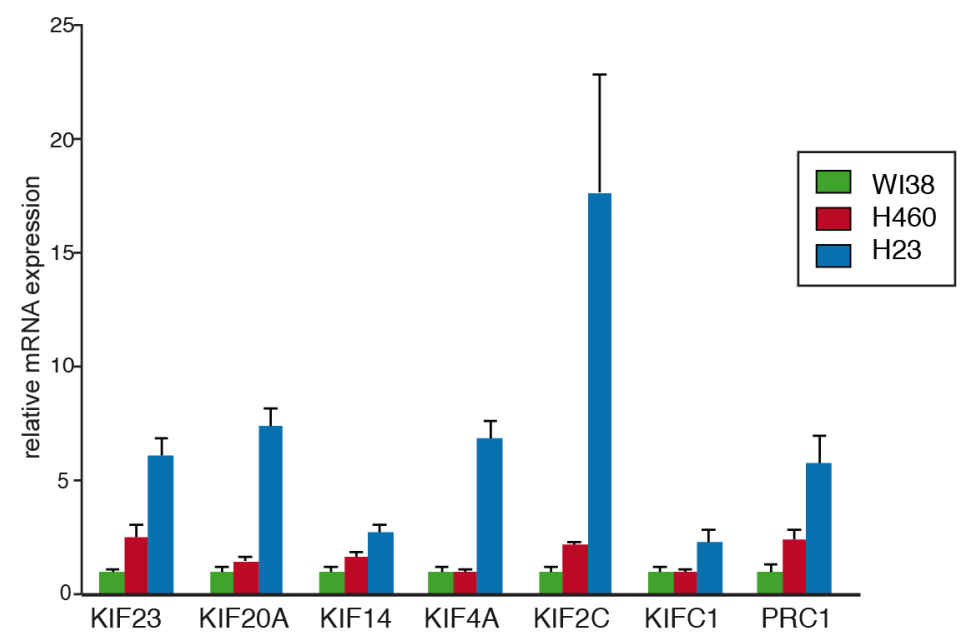

Figure 1: Mitotic kinesins and the microtubule-associated protein PRC1 are overexpressed in human lung cancer cell lines. (A) Expression of the indicated proteins were analyzed in lung cancer cell lines and in untransformed control cells (BJ, WI-38) by immunoblotting. The asterisk indicates a background band. (B) mRNA expression of kinesins and PRC1 in WI-38, H460 and H23 cells was analysed by RT-qPCR. 


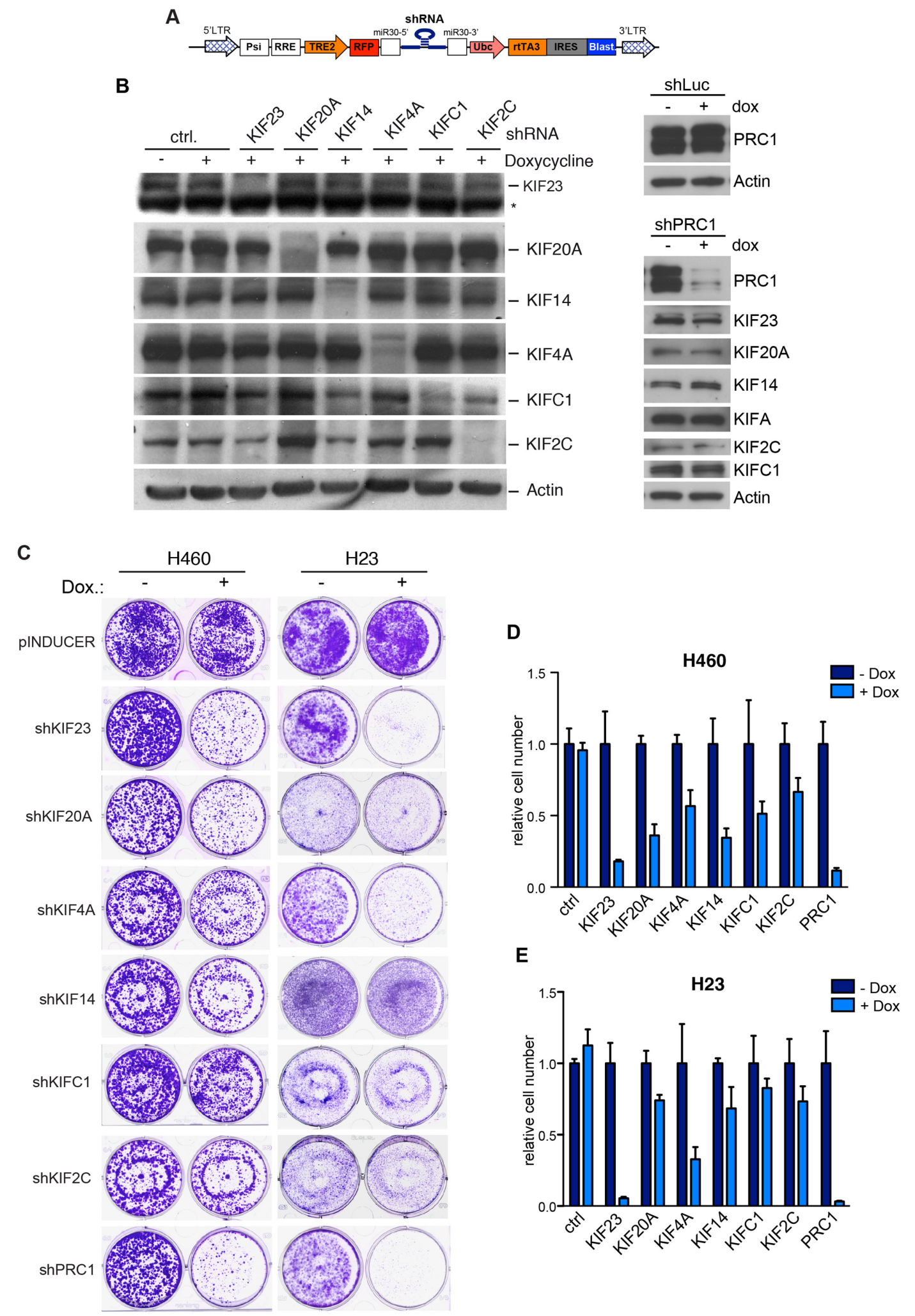

Figure 2: Mitotic kinesins and PRC1 are required for proliferation of NSCLC cells. (A) Scheme of the pINDUCER vector (B) Inducible shRNAs directed at mitotic kinesins or PRC1 were stably introduced into H23 NSCLC cells. Knockdown after induction of the shRNA with doxycycline was determined by immunoblotting. The asterisk indicates a background band. (C) H23 and H460 were cells stably transduced with the indicated inducible shRNA constructs and were plated at low density. Colony formation in absence and presence of doxycycline (to induce the shRNA) was determined by crystal violet staining after 7 days. (D and $\mathbf{E}$ ) Quantification of the colony formation assays shown in C. 
A

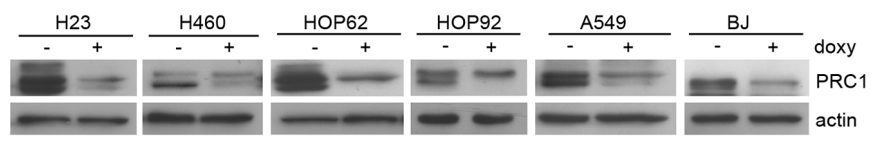

C
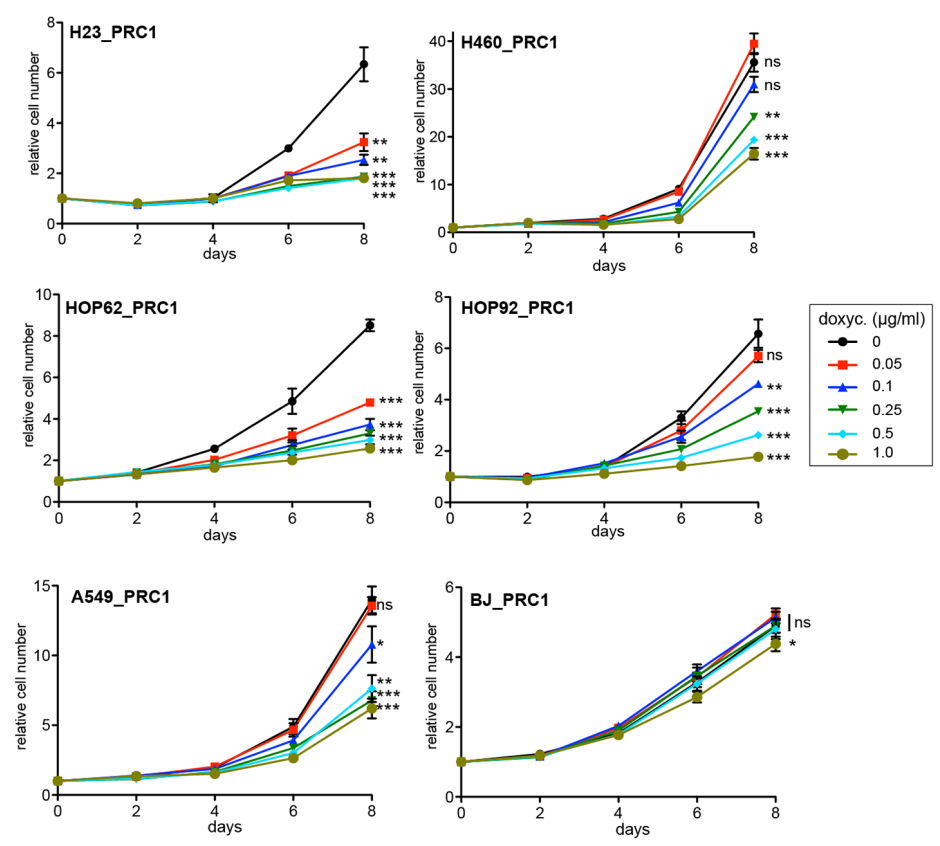

$\mathbf{E}$
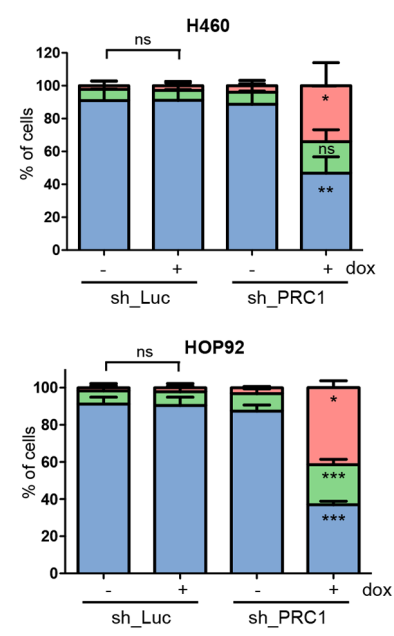
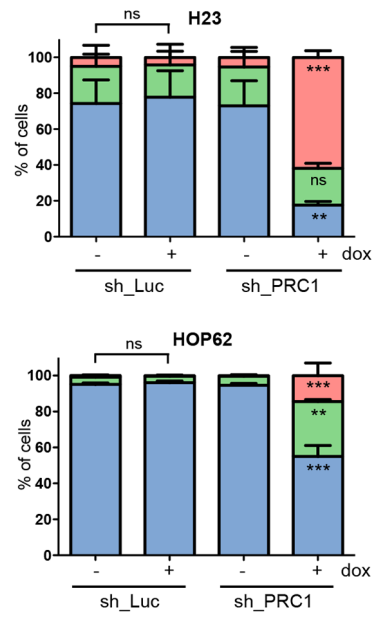

B
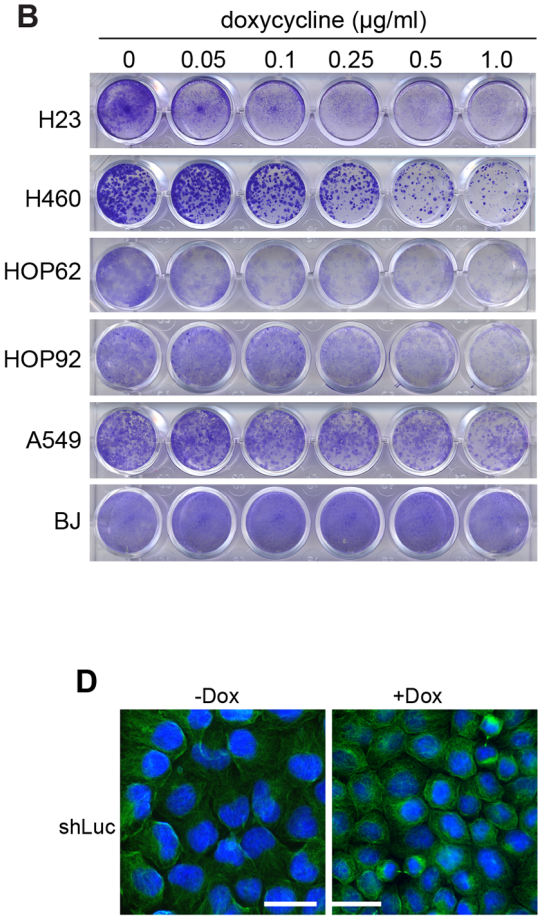

ShPRC1
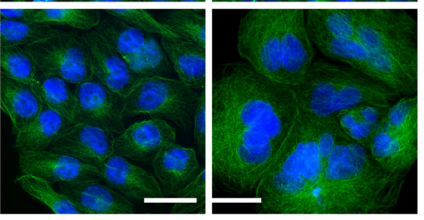

Figure 3: PRC1 is required for proliferation of lung cancer cell lines. (A) The indicated cell lines were stably infected with a PRC1-specific shRNA. Cell lines were treated with $1 \mu \mathrm{g} / \mathrm{ml}$ doxycycline for 4 days to induce the shRNA. PRC1 levels were determined by immunoblotting. (B) Cell lines expressing the PRC1-specific shRNA were cultured for a period of 8 days with the indicated concentrations of doxycycline. Colonies were stained with crystal violet. (C) Quantification of growth of lung cancer cell lines and BJ cells stably expressing the PRC1-specific shRNAs in the presence of the indicated concentrations of doxycycline was analyzed over 8 days. $\mathrm{n}=3$ replicates. Statistical significance (Student's t-test) for clarity only shown for day $8 .{ }^{*} \mathrm{p}<0.05 ;{ }^{* *} \mathrm{p}<0.001$; ${ }^{* * *} \mathrm{p}<0.0001$; ns: not significant. (D) H460 cells either expressing an inducible control shRNAs (shLuc) or a shRNA direct at PRC1 (shPRC1) were treated with or without doxycycline for 4 days. Subsequently cells were fixed and immunostained with tubulin (green) and Hoechst (blue) and investigated by fluorescence microscopy. Bar: $25 \mu \mathrm{m}$. (E) Quantification of mono-, bi- and multinucleated cells in cell lines expressing either a control shRNA (sh_Luc) or a PRC1-specific shRNA (sh_PRC1) and treated without or with $1 \mu \mathrm{g} / \mathrm{ml}$ doxycycline for 4 days to induce the PRC1 shRNA. $\mathrm{n}=\overline{3}$ replicates. Statistical significance determined by Students's t-test. ${ }^{*} \mathrm{p}<0.05 ;{ }^{* *} \mathrm{p}<0.001 ;{ }^{* * *} \mathrm{p}<0.0001$; ns: not significant. 
points was determined by crystal violet staining (Figure 3B). Quantification showed that PRC1 inhibits the proliferation of all five lung cancer cell lines in a dosedependent manner (Figure 3C). Importantly, doxycycline did not inhibit proliferation of lung cancer cell lines stably infected with the control shRNA (Supplementary Figure 2A, B). Significantly, non-tumorigenic BJ cells were less sensitive to the inhibition of PRC1 compared to the tumor cell lines. Examination by microscopy and quantification showed that silencing of PRC1 strongly increased the number of bi- and multinucleated cells in all five lung cancer cell lines, consistent with its known function in mitosis and cytokinesis (Figure 3D, 3E). Doxycycline had no effect on nucleation in cells expressing a luciferase control shRNA (Figure 3D, 3E). Although depletion of PRC1 in BJ cells also resulted in an increased fraction of binucleated cells, this effect was less pronounced compared to the tumor cell lines. Cell cycle analysis by FACS revealed a strong increase in the sub G1 population, indicative of degraded DNA, a hallmark of apoptosis, in H23 and HOP62 cells upon depletion of PRC1 (Figure 4A; Supplementary Figure 3). The sub G1 population was also increased in HOP92 cells. Immunoblot analysis of cleaved caspase 3, the effector caspase downstream of intrinsic and extrinsic apoptosis stimuli, confirmed induction of apoptosis in HOP62, HOP92 and $\mathrm{H} 23$ cells upon depletion of PRC1 (Figure 4B). Furthermore, Annexin V staining independently confirmed apoptosis upon depletion of PRC1 in HP62, HOP92 and H23 cells, but not in BJ cells. (Supplementary Figure 4). In conclusion, the depletion of PRC1 resulted in apoptosis in the three p53mutant lung cancer cell lines H23, HOP62 and HOP92. Interestingly, no apoptosis was observed in BJ cells and in A549 and H460 cells with wildtype p53-status. Instead, after depletion of PRC1, A549 and H460 cells became senescent, as evidenced by staining for ß-galactosidase, a marker of senescent cells (Figure 4C). Importantly, p53 is indeed induced by the depletion of PRC1 in p53-proficient A549 and H460 cells as shown by immunoblot analysis (Figure 4D). Furthermore, p21, a cyclin dependent kinase inhibitor and a bona-fide downstream target of p53 was also induced, indicating that p53 activity is stimulated after depletion of PRC1.

Recently, it has been reported that expression of PRC1 is induced by Wnt in hepatocellular carcinoma (HCC) cell lines [24]. Furthermore, it has been shown that PCR1 activates Wnt/ß-catenin signaling by stabilization of B-catenin [24]. Another recent study reported a similar dependency of Wnt/Bcatenin target genes on PRC1 in lung cancer cell lines [25]. We therefore tested whether $\beta$-catenin signalling is affected by the depletion of PRC1. However, acute depletion of PRC1 by the addition of doxycycline had no effect on expression of the $\beta$-catenin target gene BIRC5 in lung cancer cell lines or on a $\beta$-catenin-dependent luciferase reporter gene (Supplementary Figure 5A, B). Furthermore, Wnt3a ligand failed to induce the expression of PRC1 in $\mathrm{H} 23$ or A549 cells (Supplementary Figure 5C). In conclusion, our data do not support PRC1 as a target or regulator of $\mathrm{Wnt} /$ /-catenin signalling in lung cancer cell lines.

\section{Depletion of PRC1 inhibits anchorage- independent growth in vitro}

We next tested whether inhibition of PRC1 inhibits oncogenic transformation. To do so, we first determined the ability to grow independently of anchorage in soft agar. Control treated HOP92 cells readily formed a large number of colonies in soft agar when seed at low density (Figure 5A). Depletion of PRC1 by doxycycline resulted in a significant decrease in colony formation in soft agar. The size of the colonies was also significantly reduced following depletion of PRC1. Reduced anchorage independent growth upon depletion of PRC1 was also observed on A549 cells (Figure 5B). Taken together these data indicate that PRC1 is required for anchorageindependent growth.

\section{A requirement for $\mathrm{PRC1}$ for lung tumorigenesis in vivo}

In order to test whether PRC1 is required for tumorigenesis in vivo, we first designed several shRNAs directed at mouse PRC1. shRNAs were cloned into the inducible lentiviral pINDUCER vector and stable NIH3T3 cells were generated by lentiviral infection and selection. PRC1 levels in absence and presence of doxycycline were evaluated by immunoblotting. As shown in Figure 6A, two shRNAs resulted in efficient knockdown of PRC1 in murine cells. shRNA\#16 was cloned into a bifunctional lentiviral vector (Figure 6B). Lentiviral supernatant was produced and the virus titer was determined. Ten $\mathrm{K}-\mathrm{Ras}^{\mathrm{G} 12 \mathrm{D}} ; \mathrm{p} 53^{\mathrm{fl} / \mathrm{fl}}$ mice were infected with this virus to simultaneously activate K-Ras and delete p53 by Cre and deplete PRC1 by RNAi (Figure 6C). In parallel, ten control K-Ras ${ }^{\mathrm{G} 12 \mathrm{D}}$; $\mathrm{p} 53^{\mathrm{fl} / \mathrm{fl}}$ mice were infected with a lentivirus expressing a non-specific shRNA. To evaluate lung tumorigenesis, mice were scarified 16 weeks after tumor induction. Lung tumorigenesis was evaluated by histopathology. Importantly, the tumor area in mice infected with the lentivirus expressing the PRC1-specific shRNA was significantly smaller compared to mice infected with the virus expressing the control shRNA, indicating a requirement for PRC1 in K-RAS-driven non-small cell lung cancer in vivo (Figure 6E, 6F). Next, we asked whether expression of PRC1 is of any clinical significance for human lung cancer by examining previously published microarray data sets.

Strikingly, lung adenocarcinoma patients with tumors with high expression PRC1 showed a significant shorter survival compared to patients with low expression 
A

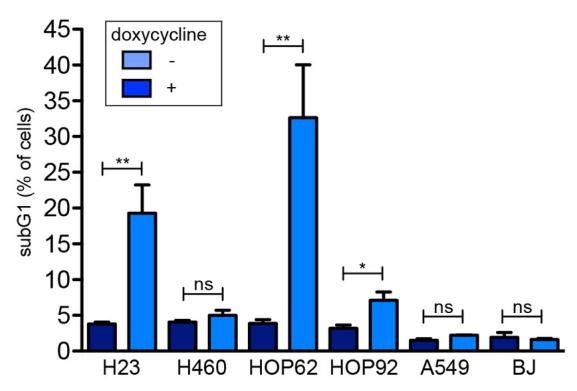

B

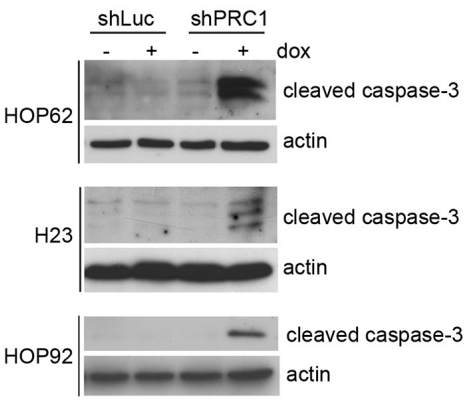

C
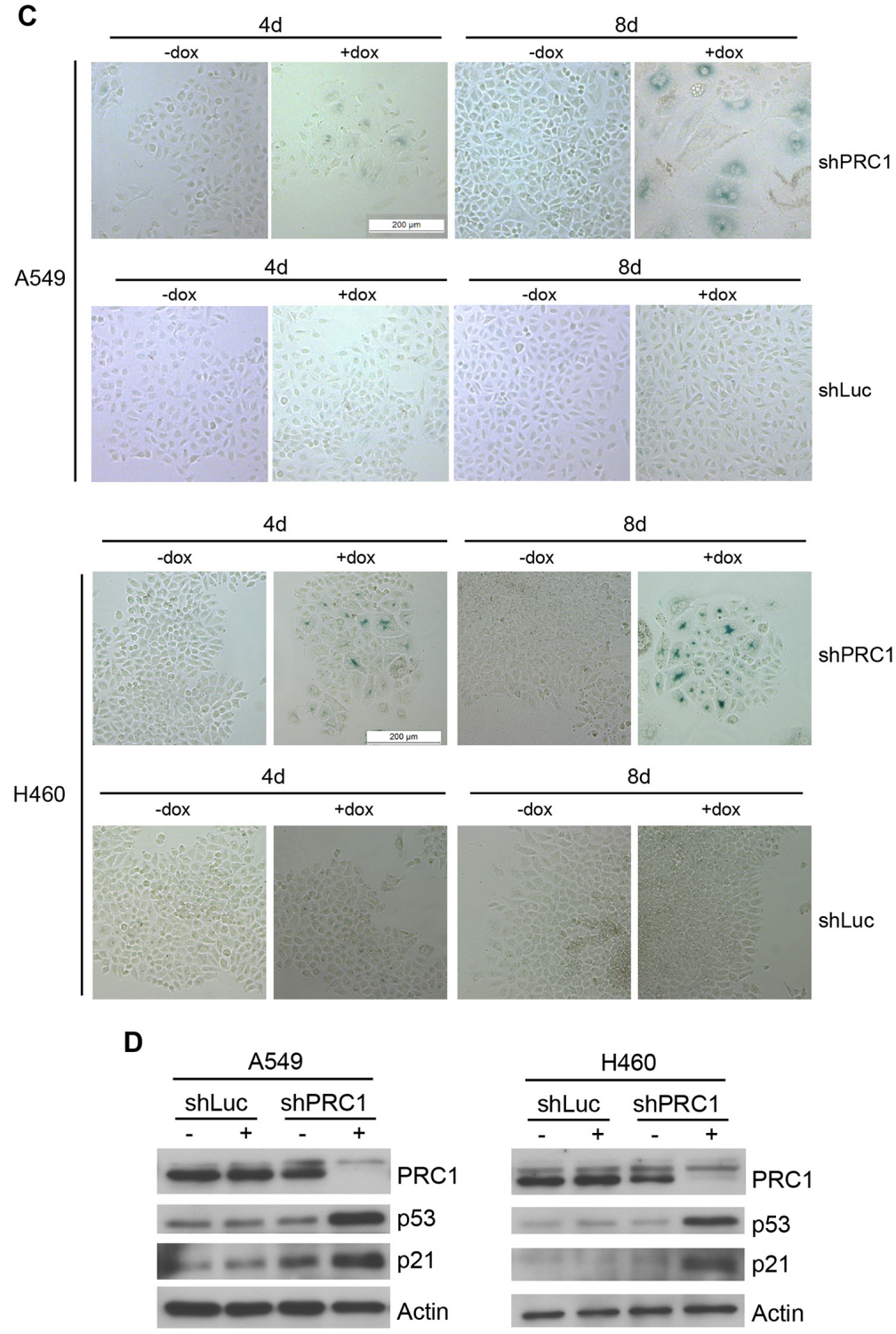

Figure 4: Depletion of PRC1 results in apoptosis in p53-mutant lung cancer cell lines and in senescence in A549 cells with $\mathbf{p 5 3}$ wildtype status. (A) The indicated cell lines stably expressing the inducible shRNA directed at PRC1 were treated with $0.5 \mu \mathrm{g} /$ $\mathrm{ml}$ doxycycline. The fraction of apoptotic subG1 cells after depletion of PRC1 was determined by FACS ( $\mathrm{n}=3$ experiments). ${ }^{* *} p<0.01,{ }^{*} \mathrm{p}$ $<0.05$. Student's $t$-test. (B) Immunoblotting for cleaved caspase-3 confirmed the induction of apoptosis in HOP62, HOP92 and H23 cells upon depletion of PRC1 with $0.5 \mu \mathrm{g} / \mathrm{ml}$ doxycycline for 8 days. (C) A549 and H460 cells stably expressing an inducible shRNA directed at PRC1 (shPRC1) or against luciferase (shLuc) were treated with $1 \mu \mathrm{g} / \mathrm{ml}$ doxycycline for 4 days or with $0.5 \mu \mathrm{g} / \mathrm{ml} \mathrm{doxycycline} 8$ days. Senescent cells were detected by staining for B-galactosidase. (D) Immunoblotting indicates that p53 and p21 are induced in A549 and $\mathrm{H} 460$ cells upon depletion of PRC1 with $1 \mu \mathrm{g} / \mathrm{ml}$ doxycycline for 4 days. 
(Figure 6G). In conclusion, we found that PRC1 is required for lung tumorigenesis in vitro and in vivo.

\section{DISCUSSION}

In this study, we investigated whether proteins that are involved in cytokinesis are potential targets for therapy of lung cancer. We found that mitotic kinesins and PRC1 are expressed at higher levels in lung cancer cell lines compared to control cell lines. Previous studies have shown that expression of mitotic kinesins is under control of the MuvB complex, B-MYB and FOXM1 $[23,26]$. MuvB is the core of an evolutionary conserved complex that regulates gene expression during the cell cycle [27]. In S- and G2-phase MuvB associates with
B-MYB and FOXM1 to activate genes required for mitosis and cytokinesis. Importantly, Myb-MuvB is under negative control by $\mathrm{p} 53$. The induction of $\mathrm{p} 53$ results in displacement of B-MYB from MMB and in formation the DREAM-p130 complex that acts as gene repressor [28]. Conversely, in the absence of p53, Myb-MuvB is hyperactive, which can explain high levels of mitotic kinesins and PRC1 in lung cancer cell lines with p53mutant status.

We find that proliferation of the H460 human lung cancer cell line with wildtype p53 status is inhibited by depletion of the investigated mitotic kinesins or by depletion of PRC1. In contrast, proliferation of $\mathrm{H} 23$ lung cancer cells which harbour a missense mutation of p53 was only suppressed by depletion of KIF23, PRC1 or

\section{A}

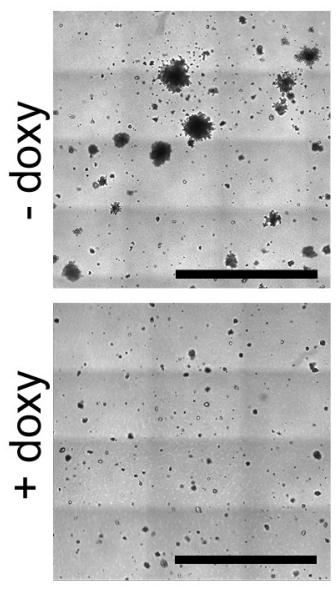

B

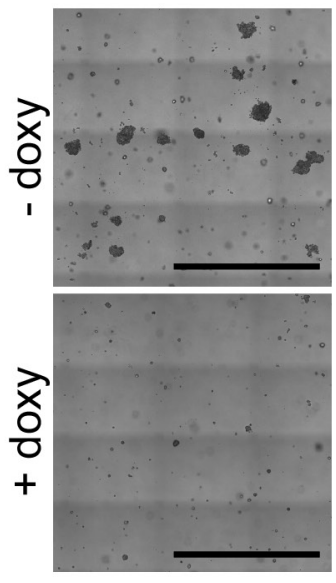

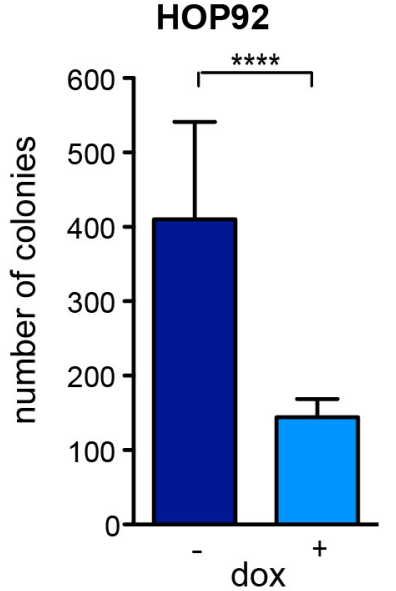

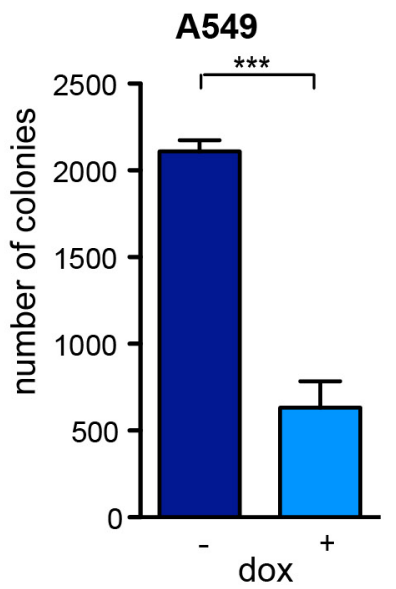

Figure 5: PRC1 is required for anchorage-independent growth. (A) HOP92 cells stably expressing the inducible PRC1 shRNA were untreated or treated with $0.5 \mu \mathrm{g} / \mathrm{ml}$ doxycycline and anchorage independent growth was analyzed in soft agar after 14 days. Left: Example pictures, bar: $2.5 \mathrm{~mm}$. Right: Quantification of colonies. $\mathrm{n}=4$ experiments. (B) Soft agar assay in A549 cells. Left: Example pictures, bar: $2.5 \mathrm{~mm}$. Right: Quantification after 18 days. $\mathrm{n}=3$ experiments. ${ }^{* * * *} p<0.0001,{ }^{* * *} p<0.001$. Student's $t$-test. 

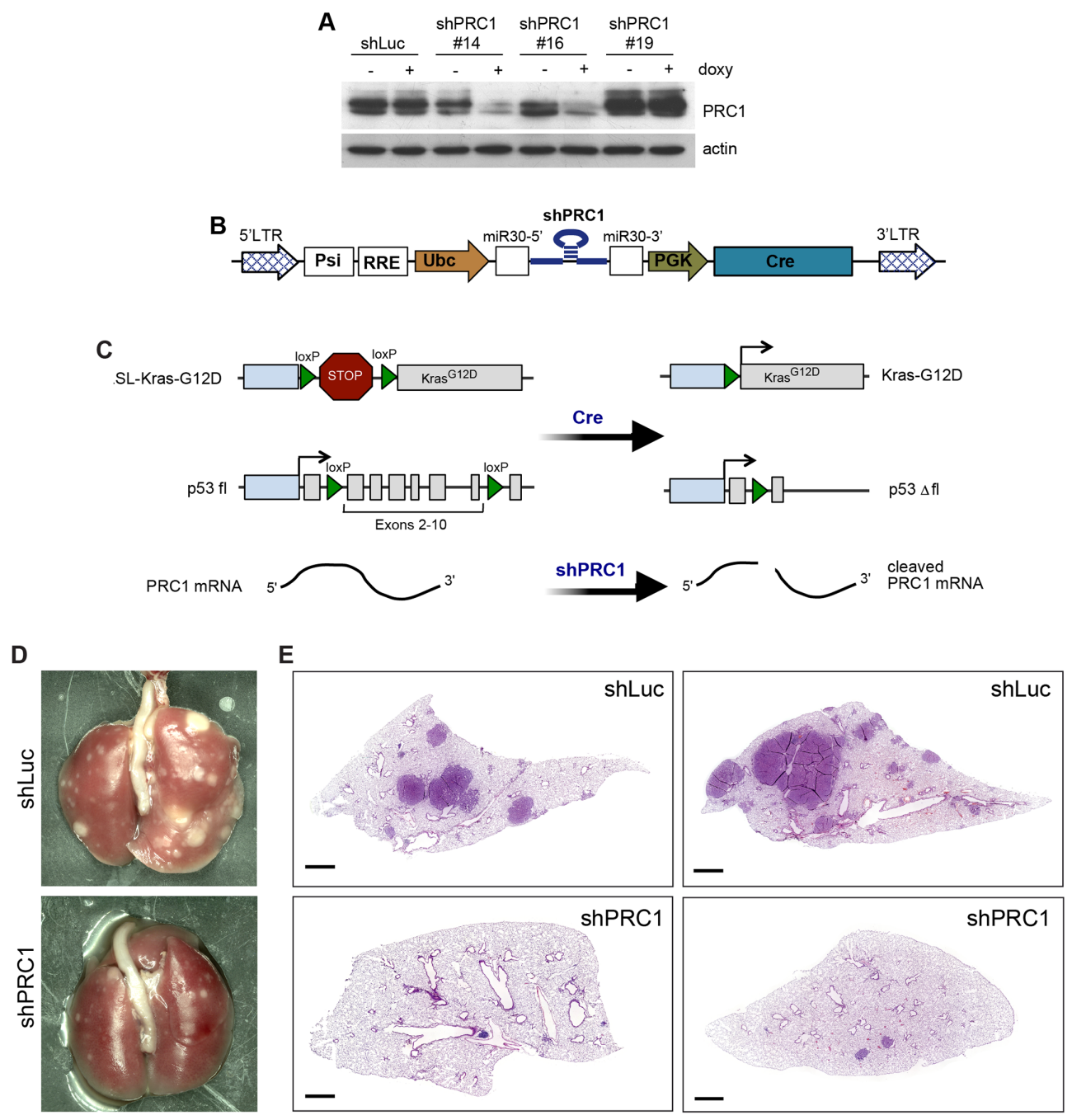

$\mathbf{F}$

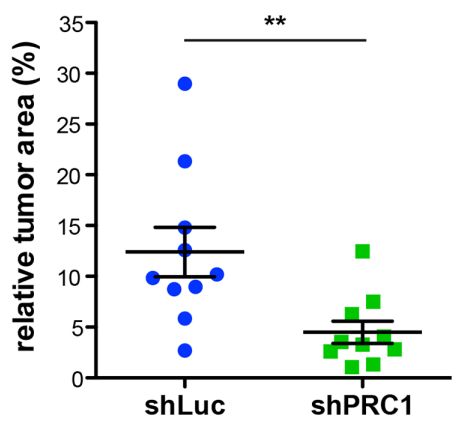

$\mathbf{G}$

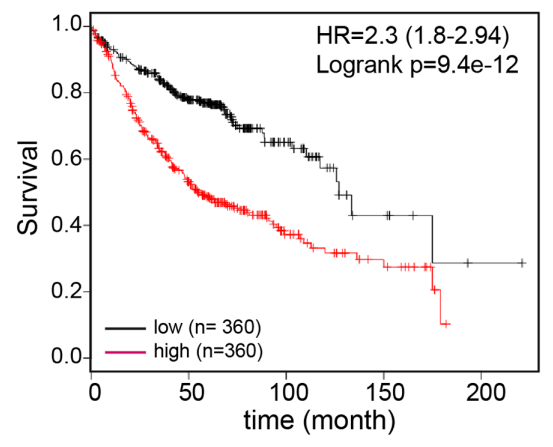

Figure 6: PRC1 is required for lung tumorigenesis in vivo. (A) Lentiviral constructs encoding shRNAs directed at murine PRC1 were introduced into NIH-3T3 cells. The depletion of PRC1 after addition of $1 \mu \mathrm{g} / \mathrm{ml}$ doxycycline for 4 days was analyzed by immunoblotting. shLuc: control shRNA directed at luciferase. (B) Scheme of the lentiviral Cre/ shRNA vector to deliver a mir30 based shRNA and Cre recombinase in vivo. (C) Strategy to activate K-Ras and delete p53 by Cre and deplete PRC1 by RNAi in a lung-specific manner by intratracheal infection of LSL-KRas ${ }^{\mathrm{G} 12 \mathrm{D}}, \mathrm{p} 3^{\mathrm{fl} / \mathrm{fl}}$ mice with the lentivirus. (D and E) LSL-KRas ${ }^{\mathrm{G} 12 \mathrm{D}} ; \mathrm{p} 53^{\mathrm{fl} / \mathrm{fl}}$ mice were infected with a bifunctional lentivirus encoding Cre and a control shRNA $(n=10)$ or with a virus encoding Cre and a shRNA specific for PRC1 ( $\mathrm{n}=10)$. Representative lungs (D) and H\&E stained lung sections are shown (E). Bar: 1mm. (F) Quantification of tumor area to total lung area. Each symbol represents the analysis of an individual animal. The tumor area was obtained by measuring all tumors from two sections from each mouse. Mean and SEM. ${ }^{* *}$ p $<0.005$ (Student's t-test.) (G) Expression of PRC1 linked to clinical outcome of lung cancer patients. KaplanMeier plot showing overall survival of lung adenocarcinoma patients based on expression of PRC1. 
KIF4A. While depletion of KIF23 and PRC1 inhibited proliferation of additional lung cancer cell lines with mutated p53, KIF4A depletion had only moderate effects in these cells. Thus, although KIF20A, KIF14, KIF4A, $\mathrm{KIFC} 1$ and KIF2C have been proposed as novel targets for cancer therapy, our data suggest that they might not be very effective in p53-mutant lung cancer cells.

The requirement of lung cancer cell lines for KIF23 is consistent our recent finding that KIF23 is necessary for lung tumor initiation in vivo [23]. In this study, we now report a similar dependency of lung tumorigenesis on PRC1, consistent with previous studies that have provided evidence for a role of PRC1 in different types of cancer [20, 24, 25]. Significantly, we observe that PRC1 is required for tumor formation in vivo in an advanced mouse model of lung adenocarcinoma induced by oncogenic K-RAS and loss of p53. Mechanistically, we provide evidence that depletion of PRC1 in lung cancer cells results in accumulation of bi- and multinucleated cells, consistent with its role as key player of central spindle organization which is essential for cytokinesis [17]. Ultimately the depletion of PRC1 caused apoptosis and senescence, which likely explains the strong inhibition of proliferation upon depletion of PRC1.

Recently, a link between PRC1 and Wnt/ßcatenin signaling in hepatocellular carcinoma (HCC) has been observed [24]. Specifically, it has been shown that the expression of PRC1 is activated by Wnt in HCC cell lines. Secondly, it has been proposed that PCR1 activates the Wnt/ß-catenin pathway by sequestration of the destruction complex and stabilization of cytoplasmic ß-catenin, suggesting that PRC1 regulates Wnt signaling in a positive feedback loop [24]. The activation of Wnt target genes by PRC1 promotes proliferation, stemness and metastasis of hepatocellular carcinoma. Interestingly, a recent study suggested a similar regulation of $\mathrm{Wnt} / \mathrm{\beta}$ catenin signaling by PRC1 in lung cancer cell lines [25]. However, our data reported here do not support a role for PRC1 in Wnt/ß-catenin signalling in lung cancer cells. First, we find that PRC1 is not induced by Wnt3a in lung cancer cell lines. Secondly, the depletion of PRC1 has no effect on the expression of a Wnt-target gene or on the activity of a ß-catenin-dependent luciferase reporter gene. The discrepancy between our findings and the results reported by Zhan et al. may be explained by differences in the experimental design. While we used a doxycyclineinducible shRNA to acutely deplete PRC1 in lung cancer cell lines for 24 hours to 3 days, Zhan et al. used noninducible lentiviral shRNAs to deplete PRC1 for 4 to 6 days. Thus, the observed effects of PRC1 on Wnt/ßcatenin signaling could be an indirect consequence of impaired cytokinesis and proliferation after a prolonged knockdown of PRC1. Instead we suggest that the dependency of lung cancer cell lines on PRC1 is a consequence of its requirement for central spindle formation and cytokinesis. Consistent with this is the increase of bi- and multinucleated cells after depletion of PRC1 as consequence of cytokinesis failure. Whether PRC1 has additional functions independent from regulation of mitosis and cytokinesis in lung cancer cells remains to be shown.

Interestingly, PRC1 is associated with a signature of 25 genes whose elevated expression is associated with chromosomal instability (CIN) and a poor prognosis in multiple cancer types [29]. It is therefore possible that the aberrant expression of PRC1 in cancer cells leads to defects in mitotic exit, thereby contributing to CIN and aneuploidy, which can trigger tumorigenesis [30]. By this mechanism, the overexpression of PRC1 could facilitate tumor initiation.

In conclusion, PRC1 could represent a novel target for therapy of lung cancer. Due to its specialized function in cytokinesis, its inhibition might produce fewer side effects than drugs that directly target tubulin.

\section{MATERIALS AND METHODS}

\section{Mice}

All animals were maintained on a C57BL/6 background. $L S L-K-R A S^{G 12 D}$ and mice carrying a conditional allele of $p 53$ have been described [31, 32]. $L S L-K-R A S^{G 12 D}$ and conditional p53 mice were obtained from the NCI Mouse Repository, Bethesda, Maryland. Tumors were initiated in 9-15 weeks old male and female mice by intratracheal infection with a lentiviral vector expressing Cre-recombinase $\left(10^{6}\right.$ lentiviruses per mouse) as described previously [33]. Sample size was based on comparable studies in the literature. To deliver the virus, mice were anesthetized with Ketamin/ Xylazin. For infection with the Cre-shLuc (control) or Cre-shPRC1 lentivirus, $L S L-K-R A S^{G 12 D} ; p 53^{\text {fllfl mice were }}$ randomly allocated to 2 groups. All animal experiments were carried out according to protocols that were approved by an institutional committee (Tierschutzkommission der Regierung von Unterfranken).

\section{Histopathology}

Mouse lung tissues were fixed in 4\% paraformaldehyde in phosphate buffered saline (PBS), embedded in paraffin and sectioned. Sections were deparaffinized, rehydrated and stained with hematoxylin and eosin.

\section{Lentivirus production and titer determination}

Lentviruses were produced as described previously [34]. Briefly, a lentiviral pINDUCER construct containing a shRNA directed at mitotic kinesins or at PRC1 was cotransfected together with psPAX2 and VSVg plasmid into 293 T cells. 48 hours after transfection the lentiviral 
supernatant was collected and used to infect human lung cancer cell lines. The lentiviral pINDUCER vectors for inducible expression of shRNAs directed at kinesins and PRC1 have been described [26].

For the in vivo experiments a bifunctional pINDUCER vector encoding for Cre-recombinase and a shRNA specific for murine PRC1 was generated. The biological lentiviral titer was determined by infecting a reported cell line that expresses GFP after Cre-mediated recombination [23]. The fraction of GFP-positive cells after lentivirus infection was determined by flow cytometry and the lentivirus titer was calculated.

\section{Cell culture}

H23, H460, HOP62, HOP92, H226, H522, H358, H441 and A549 cells were cultured in RPMI containing $10 \%$ FCS (Invitrogen). BJ cells were cultured in DMEM (Invitrogen) containing 10\% FCS (Invitrogen).

\section{Antibodies}

The following primary antibodies were used: Actin: sc-47778 (Santa Cruz Biotechnology). KIF4A: A301-074A (Bethyl Laboratories). PRC1: sc-8356 (Santa Cruz Biotechnology). KIF14: A300-912A (Bethyl Laboratories). KIF20A: A300-879A/Bethyl Laboratories). KIF23: sc-867 (Santa Cruz Biotechnology). KIF2C: kind gift from Linda Wordeman. KIFC1: 12313 (Cell Signalling). a-tubulin (Sigma T6074).

\section{Immunoblotting}

Cells were lysed in TNN $[50 \mathrm{mM}$ Tris $(\mathrm{pH}$ 7.5), $120 \mathrm{mM} \mathrm{NaCl}, 5 \mathrm{mM}$ EDTA, 0.5\% NP40, 10 $\mathrm{mM} \mathrm{Na} \mathrm{P}_{2} \mathrm{O}_{7}, 2 \mathrm{mM} \mathrm{Na} \mathrm{VO}_{4}, 100 \mathrm{mM} \mathrm{NaF}, 10 \mathrm{mg} /$ $\mathrm{mL}$ phenylmethylsulfonyl fluoride, protease inhibitors (Sigma)]. Proteins were separated by SDS-PAGE, transferred to PVDF membrane and detected by immunoblotting.

\section{Flow cytometry}

For flow cytometry, cells were fixed in $80 \%$ ethanol, incubated in $38 \mathrm{mM}$ sodium citrate and $500 \mu \mathrm{g} / \mathrm{ml}$ RNase A for $30 \mathrm{~min}$ at $37^{\circ} \mathrm{C}$ and stained with $43 \mathrm{mM}$ propidium iodide. Samples were analyzed on a Beckman Coulter Fc500.

\section{Proliferation assays}

For growth curves, cells were plated in 24-well plates and expression of the shRNA was induced with the indicated concentrations of doxycycline. The following cell numbers were plated (per well of a 24-well plate): 1,500 cells (A549), 1,000 cells (HOP92), 1,500 cells (H23), 1,000 cells (H460), 2000 cells (HOP62), 5000 cells
(BJ). At the indicated time points, cells were fixed in $10 \%$ formaline and stained with $0.1 \%$ crystal violet. The dye was extracted with $10 \%$ acetic acid and the optical density was determined as described [35].

\section{Soft agar assay}

For soft agar, cells were resuspended in $0.35 \%$ low melting agarose in soft agar medium [DMEM, 3.7\% 1M sodium bicarbonate, 20\% FCS, $20 \mathrm{mM}$ glutamax, $9 \mathrm{mg} /$ ml D-glucose, 1\% PenStrep]. 10,000 cells were seeded on a base layer of $0.7 \%$ low melting agarose in soft agar medium in a 6-well plate. For induction of the shRNA, $0.5 \mu \mathrm{g} / \mathrm{ml}$ doxycycline was added directly to the soft agar medium. Cells were cultivated for 14- or 18 days and fed every 3 days. Colony formation was analyzed by microscopy.

\section{Annexin V staining}

Detection of apoptotic cells by Annexin V-FITC staining was performed as recommended by the manufacturer (Thermo Fisher). Briefly, cells were cultured in 24-well plates were washed twice with PBS and stained with Annexin V-FITC for $10 \mathrm{~min}$ in the dark. Detection of Annexin V-FITC were performed with a fluorescence microscope after two washing steps with binding buffer.

\section{Senescence-associated $\beta$-galactosidase staining}

Senescence-associated $\beta$-galactosidase activity was detected as described [36]. Briefly, cells were fixed with $3.7 \%$ formaldehyde in PBS for $5 \mathrm{~min}$ and washed twice with PBS. X-gal staining solution [1 mg/ml X-Gal, 40 $\mathrm{mM}$ citric acid/sodium phosphate buffer (dibasic, each $200 \mathrm{mM}), 5 \mathrm{mM}$ potassium ferrocyanide $(\mathrm{K} 4 \mathrm{Fe}(\mathrm{CN}) 6)$, $5 \mathrm{mM}$ potassium ferricyanide $(\mathrm{K} 3 \mathrm{Fe}(\mathrm{CN}) 6), 150 \mathrm{mM}$ $\mathrm{NaCl}, 2 \mathrm{mM} \mathrm{MgCl}$ ] was added to the cells. Cells were incubated at $37^{\circ} \mathrm{C}$ and $5 \% \mathrm{CO} 2$ for $16 \mathrm{~h}$ protected from light and then washed twice with PBS. The results were documented by microscopy.

\section{Immunostaining}

Cells were fixed in 3\% paraformaldehyde, $2 \%$ sucrose in PBS, permeabilized with $0.2 \%$ Triton X-100 and blocked with $3 \%$ BSA for 30 minutes. Coverslips were incubated for 1 hour at room temperature with ant-tubulin antibody. Secondary antibody (a-mouse Alexa Fluor 488 and Alexa Fluor 700, Thermo Fischer) was diluted 1:500 in PBS and incubated $2 \mathrm{~h}$ at room temperature. Coverslips were stained with $1 \mu \mathrm{g} / \mathrm{ml} \mathrm{4}$,,6-diamidino-2-phenylindole (Hoechst; Sigma) and mounted in IMMU-MOUNT. Cells were investigated by fluorescence microscopy and the fraction of mono- and multinucleated cells was determined. 


\section{RNA isolation, reverse transcription and quantitative real-time PCR}

Total RNA was isolated with Total RNA Isolation Reagent according to the manufacturer's protocol (Thermo Scientific). $2 \mu \mathrm{g}$ RNA was transcribed using 125 units MMuLv (Thermo Scientific). Quantitative real-time PCR reagents were from Thermo Scientific and realtime PCR was performed using the Mx3000 (Stratagene) detection system. Expression differences were calculated as described before [37].

\section{Luciferase assay}

Cells were transfected with TOPflash (three wildtype TCF4 binding sites) or with FOPflash (three mutated TCF4 binding sites) and as a reference with a Renilla expressing construct. After treatment with doxycycline cells were lysed in 1x passive laysis buffer (Promega). Lysates were mixed with a ATP working solution (20 mM ATP, $25 \mathrm{mM}$ Gly-Gly pH 7,8, 1M $\mathrm{MgSO} 4$ ) for measurement of the luciferase signal or used directly for measurement of the Renilla signal. Luciferase signal or Renilla signal were measured with a luminescence microplate reader (TriStar LB941 Berthold Technologies) and primed in advance either with a luciferin working solution (1 mM luciferin, $25 \mathrm{mM}$ GlyGly $\mathrm{pH} 7,8)$ or with a renilla assay buffer $(1 \mathrm{M} \mathrm{NaCl}, 2$ mM EDTA-Na2, 0,22 M potassium phosphate dibasic, 3 $\mathrm{mM} \mathrm{NaN} 3,0,44 \mathrm{mg} / \mathrm{ml} \mathrm{BSA}, 43 \mu \mathrm{M}$ coelenterazine).

\section{Statistical analysis}

Statistical analyses were performed using Prism 5 (GraphPad Software). Statistical significance was determined using Student's t-test. $\mathrm{P}$ values $<0.05$ were considered statistically significant.

\section{Survival analysis}

Survival analyses and gene expression correlation analysis for human lung cancer patients was performed using the KM-plotter (http://kmplot.com/analysis) [38].

\section{ACKNOWLEDGMENTS}

We thank all members of the laboratory for their suggestions and critical reading of the manuscript. We thank Susi Spahr for her help in preparing histological sections.

\section{CONFLICTS OF INTEREST}

The authors declare no conflicts of interest.

\section{GRANT SUPPORT}

This work was supported by grants from the Deutsche Krebshilfe (110928) and Sander Stiftung (2015.038.1) towards SG.

\section{REFERENCES}

1. Jemal A, Bray F, Center MM, Ferlay J, Ward E, Forman D. Global cancer statistics. CA Cancer J Clin. 2011; 61:69-90.

2. Riely GJ, Marks J, Pao W. KRAS mutations in non-small cell lung cancer. Proc Am Thorac Soc. 2009; 15;6:201-5.

3. Cox AD, Fesik SW, Kimmelman AC, Luo J, Der CJ. Drugging the undruggable RAS: mission possible? Nat Rev Drug Discov. 2014;13:828-51.

4. Engelman JA, Chen L, Tan X, Crosby K, Guimaraes AR, Upadhyay R, Maira M, McNamara K, Perera SA, Song Y, Chirieac LR, Kaur R, Lightbown A, et al. Effective use of PI3K and MEK inhibitors to treat mutant Kras G12D and PIK3CA H1047R murine lung cancers. Nat Med. 2008;14:1351-6.

5. Jokinen E, Koivunen JP. MEK and PI3K inhibition in solid tumors: rationale and evidence to date. Ther Adv Med Oncol. 2015; 7:170-80.

6. Zhu Z, Golay HG, Barbie DA. Targeting pathways downstream of KRAS in lung adenocarcinoma. Pharmacogenomics. 2014; 15:1507-18.

7. Luo J, Solimini NL, Elledge SJ. Principles of cancer therapy: oncogene and non-oncogene addiction. Cell. 2009; 136:823-37.

8. Corcoran RB, Cheng KA, Hata AN, Faber AC, Ebi H, Coffee EM, Greninger P, Brown RD, Godfrey JT, Cohoon TJ, Song Y, Lifshits E, Hung KE, et al. Engelman JA. Synthetic lethal interaction of combined BCL-XL and MEK inhibition promotes tumor regressions in KRAS mutant cancer models. Cancer Cell. 2013; 23:121-8.

9. Kumar MS, Hancock DC, Molina-Arcas M, Steckel M, East P, Diefenbacher M, Armenteros-Monterroso E, Lassailly F, Matthews N, Nye E, Stamp G, Behrens A, Downward J. The GATA2 transcriptional network is requisite for RAS oncogene-driven non-small cell lung cancer. Cell. 2012; 149:642-55.

10. Luo J, Emanuele MJ, Li D, Creighton CJ, Schlabach MR, Westbrook TF, Wong KK, Elledge SJ. A genome-wide RNAi screen identifies multiple synthetic lethal interactions with the Ras oncogene. Cell. 2009;137:835-48.

11. Malumbres M. Oncogene-induced mitotic stress: p53 and pRb get mad too. Cancer Cell. 2011; 19:691-2.

12. Dikovskaya D, Cole JJ, Mason SM, Nixon C, Karim SA, McGarry L, Clark W, Hewitt RN, Sammons MA, Zhu J, Athineos D, Leach JD, Marchesi F, et al. Mitotic stress is an integral part of the oncogene-induced senescence program that promotes multinucleation and cell cycle arrest. Cell Rep. 2015; 12:1483-96. 
13. Huang HC, Shi J, Orth JD, Mitchison TJ. Evidence that mitotic exit is a better cancer therapeutic target than spindle assembly. Cancer Cell. 2009; 16:347-58.

14. McKenzie C, D’Avino PP. Investigating cytokinesis failure as a strategy in cancer therapy. Oncotarget. 2016; 7:8732341. https://doi.org/10.18632/oncotarget.13556.

15. Lee KY, Davies T, Mishima M. Cytokinesis microtubule organisers at a glance. J Cell Sci. 2012; 125:3495-500.

16. Green RA, Paluch E, Oegema K. Cytokinesis in animal cells. Annu Rev Cell Dev Biol. 2012; 28:29-58.

17. Glotzer M. The $3 \mathrm{Ms}$ of central spindle assembly: microtubules, motors and MAPs. Nat Rev Mol Cell Biol. 2009; 10:9-20.

18. Kozielski F. Kinesins and Cancer. Springer; 2015. 1 p.

19. Cai S, Weaver LN, Ems-McClung SC, Walczak CE. Proper Organization of microtubule minus ends is needed for midzone stability and cytokinesis. Curr Biol. 2010; 20:880-5.

20. Shimo A, Nishidate T, Ohta T, Fukuda M, Nakamura Y, Katagiri T. Elevated expression of protein regulator of cytokinesis 1 , involved in the growth of breast cancer cells. Cancer Sci. 2007; 98:174-81.

21. Kwon M, Godinho SA, Chandhok NS, Ganem NJ, Azioune A, Thery M, Pellman D. Mechanisms to suppress multipolar divisions in cancer cells with extra centrosomes. Genes Dev. 2008; 22:2189-203.

22. Rath O, Kozielski F. Kinesins and cancer. Nat Rev Cancer. 2012; 12:527-39.

23. Iltzsche F, Simon K, Stopp S, Pattschull G, Francke S, Wolter P, Hauser S, Murphy DJ, García P, Rosenwald A, Gaubatz S. An important role for Myb-MuvB and its target gene KIF23 in a mouse model of lung adenocarcinoma. Oncogene. 2017; 36:110-21.

24. Chen J, Rajasekaran M, Xia H, Zhang X, Kong SN, Sekar K, Seshachalam VP, Deivasigamani A, Goh BKP, Ooi LL, Hong W, Hui KM. The microtubule-associated protein PRC1 promotes early recurrence of hepatocellular carcinoma in association with the $\mathrm{Wnt} / \beta$-catenin signalling pathway. Gut. 2016; 65:1522-34.

25. Zhan P, Zhang B, Xi GM, Wu Y, Liu HB, Liu YF, Xu WJ, Zhu QQ, Cai F, Zhou ZJ, Miu YY, Wang XX, Jin JJ, et al. $\mathrm{PRC} 1$ contributes to tumorigenesis of lung adenocarcinoma in association with the $\mathrm{Wnt} / \beta$-catenin signaling pathway. Mol Cancer. 2017; 16:108.

26. Wolter P, Hanselmann S, Pattschull G, Schruf E, Gaubatz $\mathrm{S}$. Central spindle proteins and mitotic kinesins are direct transcriptional targets of MuvB, B-MYB and FOXM1 in breast cancer cell lines and are potential targets for therapy.
Oncotarget. 2017; 8:11160-72. https://doi.org/10.18632/ oncotarget.14466.

27. Sadasivam S, DeCaprio JA. The DREAM complex: master coordinator of cell cycle-dependent gene expression. Nat Rev Cancer. 2013; 13:585-95.

28. Mannefeld M, Klassen E, Gaubatz S. B-MYB is required for recovery from the DNA damage-induced G2 checkpoint in p53 mutant cells. Cancer Res. 2009; 69:4073-80.

29. Carter SL, Eklund AC, Kohane IS, Harris LN, Szallasi Z. A signature of chromosomal instability inferred from gene expression profiles predicts clinical outcome in multiple human cancers. Nat Genet. 2006; 38:1043-8.

30. Bakhoum SF, Compton DA. Chromosomal instability and cancer: a complex relationship with therapeutic potential. J Clin Invest. 2012; 122:1138-43.

31. Jackson EL, Willis N, Mercer K, Bronson RT, Crowley D, Montoya R, Jacks T, Tuveson DA. Analysis of lung tumor initiation and progression using conditional expression of oncogenic K-ras. Genes Dev. 2001; 15:3243-8.

32. Marino S, Vooijs M, van der Gulden H, Jonkers J, Berns A. Induction of medulloblastomas in p53-null mutant mice by somatic inactivation of $\mathrm{Rb}$ in the external granular layer cells of the cerebellum. Genes Dev. 2000;14:994-1004.

33. DuPage M, Dooley AL, Jacks T. Conditional mouse lung cancer models using adenoviral or lentiviral delivery of Cre recombinase. Nat Protoc. 2009; 4:1064-72.

34. Tiscornia G, Singer O, Verma IM. Production and purification of lentiviral vectors. Nat Protoc. 2006; 1:241-5.

35. Serrano M, Lin AW, McCurrach ME, Beach D, Lowe SW. Oncogenic ras provokes premature cell senescence associated with accumulation of p53 and p16INK4a. Cell. 1997; 88:593-602.

36. Dimri GP, Lee X, Basile G, Acosta M, Scott G, Roskelley C, Medrano EE, Linskens M, Rubelj I, Pereira-Smith O. A biomarker that identifies senescent human cells in culture and in aging skin in vivo. Proc Natl Acad Sci USA. 1995; 92:9363-7.

37. Osterloh L, Eyss von B, Schmit F, Rein L, Hübner D, Samans B, Hauser S, Gaubatz S. The human synMuv-like protein LIN-9 is required for transcription of G2/M genes and for entry into mitosis. EMBO J. 2007; 26:144-57.

38. Györffy B, Lánczky A, Eklund AC, Denkert C, Budczies J, Li Q, Szallasi Z. An online survival analysis tool to rapidly assess the effect of 22,277 genes on breast cancer prognosis using microarray data of 1,809 patients. Breast Cancer Res Treat. $2010 ; 123: 725-31$. 